\title{
Perfil de salto vertical, velocidad, flexibilidad y composición corporal de porteros de balonmano en categorías inferiores \\ Jump height, velocity, flexibility and anthropometric profile of handball goalkeepers in young categories
}

Sergio Sebastia-Amat, Jose Julio Espina-Agullo y Juan Jose Chinchilla-Mira

Universidad de Alicante (España)

Resumen. El objetivo de este estudio se centra en la determinación del perfil antropométrico y condicional del portero de balonmano de categorías inferiores pertenecientes a equipos de la Federación de Balonmano de la Comunidad Valenciana.

La muestra consta de 26 porteros masculinos de balonmano de edades comprendidas entre los 11 y los 20 años. Las pruebas realizadas para el perfil antropométrico fueron altura, peso, IMC, porcentaje de grasa corporal y kilogramos de masa magra, mientras que para el perfil condicional fueron sprint 5 metros y 15 metros, sit and reach y salto vertical (SJ, CMJ y ABK). Se realizó un análisis de la varianza de un factor (ANOVA) y una prueba post-hoc (Tukey) para establecer las diferencias estadísticas entre los grupos en las diferentes variables de estudio. Los mejores resultados se produjeron en el Grupo 3 (G3), excepto en la prueba sit and reach que se produjo en el Grupo 2 (G2). La mayor diferencia se produjo entre el Grupo 1 (G1) y el Grupo 3 (G3), mostrando diferencias significativas en ocho de las once variables analizadas. Los resultados muestran que el portero de balonmano posee una edad madurativa avanzada. Las categorías de mayor edad presentan mejores resultados en los test realizados existiendo mayores diferencias entre los grupos en el perfil antropométrico que en el aspecto condicional.

Palabras clave: Balonmano, portero, valoración condicional, perfil antropométrico, categorías inferiores.

Abstract. The aim of this paper is to determinate the anthropometric and conditional profile of young handball goalkeepers that belong to teams of Valencian Handball Federation. The sample is based on an evaluation of 26 male handball goalkeepers aged between 11 to 20 years. The tests performed for the anthropometric profile were height, weight, BMI, percentage of body fat and kilograms of lean muscle mass, while for the conditional profile were 5 meters sprint and 15 meters sprint, sit and reach and vertical jump (SJ, CMJ and ABK). An inferential analysis was performed using a one-way analysis of variance (ANOVA) and a post-hoc test (Tukey) to establish the differences between groups in the different study variables. The best results occurred in group 3, except in the sit and reach test that occurred in group 2. The biggest differences were appreciated between group 1 and group 3, showing significant differences in eight of the eleven variables analyzed. The results show that the handball goalkeepers show an advanced maturity age. The older categories show better results in the tests performed with bigger differences between groups in height anthropometric profile than in the conditional aspect.

Key words: Handball, goalkeeper, physical evaluation, body composition, young players.

\section{Introducción}

Desde el punto de vista del rendimiento físico, distintos estudios consideran que el perfil antropométrico, la fuerza, la velocidad y la habilidad son considerados factores importantes en el rendimiento de los deportistas de élite (Díaz, Rojas, y Castro, 2016; Gorostiaga, Granados, Ibáñez, e Izquierdo, 2005; Iturriaga, García-Cervantes, y Lara, 2016; Mohamed et al., 2009). Por este motivo, diferentes autores han tratado la importancia del perfil antropométrico y las demandas físicas como factores del rendimiento en diferentes deportes (Zurgo, De Haro, Hemández, y Arribas, 2016) entre los que seencuentra el balonmano (Ziv y Lidor, 2009).

Concretamente, si secompara el perfil de los jugadores debalonmano con perfiles de otros deportistas en categorías inferiores podemos ver como en el estudio de Markovic, Vuckovic, Sekulic, y Gadžic (2015) los jugadores de balonmano presentaron valores más altos en la mayoría de las variables estudiadas, tanto en las antropométricas como en las de condición física, siendo esas diferencias significativas en 11 de las 15 . En el estudio de Grijota, Muñoz, Crespo, Robles, y Maynar (2012) se comparó a los jugadores de balonmano con nadadores y karatecas pertenecientes a categorías inferiores. Los jugadores de balonmano y los nadadores presentaron valores semejantes en cuanto a altura y peso aunque igual que en el estudio anterior los jugadores de balonmano presentaron porcentajes de grasa corporal elevados. También se observaron diferencias significativas entre el porcentaje graso y muscular.

Enjugadores deélite Peña, Moreno-Doutres, Coma, Cook, y Buscà (2016) realizaron un estudio en el que comparaban el perfil antropométrico y el perfil condicional de jugadores profesionales de baloncesto, balonmano y voleibol. Los resultados mostraron que los jugadores de balonmano presentaban valores bajos, sin destacar en

Fecha recepción: 15-11-16. Fecha de aceptación: 10-03-17

Sergio Sebastia-Amat

sergiosebastiaamat@gmail.com ninguna variable analizada. Al igual que en los estudios realizados en categorías inferiores, los jugadores de balonmano fueron los deportistas que mayor porcentaje de grasa corporal presentaron pudiéndose deber a las características de este deporte, el nivel de maduración, y la existencia de puestos específicos donde se requieren jugadores muy pesados (Grijota et al., 2012).

Por otro lado, en cuanto al estudio del perfil recogido en la literatura, se ha considerado importante establecer un modelo del deportista en función de su posición en el campo y de la categoría en la que compite (Haugen, Tønnessen, y Seiler, 2016).

La posición de portero es la más específica puesto que es el último defensa de su equipo y sus condiciones de manejo e interacción con la pelota son diferentes al resto de sus compañeros. Así pues, debe actuar individualmente en espacios reducidos y estar concentrado en realizar acciones simples de forma rápida y explosiva en una fracción de segundo (Milanese et al, 2011).

Espina-Agulló, Pérez-Turpin, Jiménez-Olmedo, Penichet-Tomás, y Pueo (2016) sugieren que a pesar de las modificaciones acontecidas en el balonmano en los últimos 30 años, el rol del portero en las acciones defensivas del juego ha cambiado poco.

Por otra parte, en cuanto a las demandas energéticas, no son tan exigentes respecto al resto de posiciones (Srhoj, Marinovic, y Rogulj, 2002) aunque su papel en la evolución del desarrollo del juego es realmente importante siendo el primero y el último en la primera línea de defensa al mismo tiempo que es el primer y último atacante (Dumitru, 2010).

Concretamente en adultos, el portero debe poseer una gran estatura y envergadura, ya que de ese modo, el espacio que ocupan y las posibilidades de interceptar los balones resultan mayores (Sporis, Vuleta, Vuleta, y Milanovic, 2010). Del mismo modo, los jugadores de la primera línea y los porteros son los jugadores que presentan mejores valores antropométricos tanto en categoría masculina (Chaouachi et al., 2009) como femenina (Vila et al., 2012). Lo mismo sucede en categorías inferiores donde podemos resaltar que los pivotes presentan el mayor porcentaje de grasa corporal (Matthys et al., 2013). 
Sin embargo, los estudios realizados en categorías inferiores de balonmano en los que se relaciona el perfil antropométrico y las demandas físicas son limitados (Gorostiaga, Granados, Ibáñez, e Izquierdo, 2005) y más si la información hace referencia a un puesto tan específico como es el portero.

Por tanto, el objetivo de este estudio es describir el perfil antropométrico y condicional de una muestra de porteros pertenecientes a la Federación de Balonmano de la Comunidad Valenciana, de tal forma que se pueda determinar dicho perfil en función de la categoría de edad a la que pertenezca.

\section{Método}

\section{Participantes}

Un total de 26 jugadores masculinos de edades comprendidas entre los 10 y los 20 años procedentes de distintos equipos de balonmano de la Comunidad Valenciana participaron en el estudio. Todos los jugadores ocupaban la posición de portero dentro de sus respectivos equipos.

El estudio fue aprobado por el Comité Ético de la Universidad de Alicante, contando con el consentimiento de los deportistas o tutores legales de aquellos que eranmenores de edad, informándose debidamente del procedimiento y partes del estudio.

\section{Instrumentos}

Para llevar a cabo el estudio y las medidas se empleó para peso $(0,1 \mathrm{Kg})$ y altura $(0,1 \mathrm{~cm})$ una báscula mecánica con tallímetro Astra. Para masa corporal $(0,1 \mathrm{~kg})$ masa magra $(0,1 \mathrm{~kg})$ y porcentaje de masa grasa $(0,1 \%)$ un aparato de análisis de composición corporal (Tanita BC-545N) (Markoviæ, Vuèkoviæ, Sekuliæ, y Gadžæ, 2015; Matthys et al., 2013; Nikolaidis, e Ingebrigtsen, 2013).

\section{Procedimiento}

La toma de datos se realizó durante el desarrollo del campus de porteros celebrado en la provincia de Alicante.

Los jugadores fueron divididos en tres grupos según su edad: el Grupo 1 ( $n=12$ ) estaba formado por jugadores con edades comprendidas entre 10-13 años, el Grupo $2(n=9)$ estaba formado por jugadores con edades comprendidas entre 14-16 años y el Grupo $3(n=5)$ estaba formado por jugadores con edades comprendidas entre 17-20 años.

En el estudio se llevan a cabo diferentes pruebas y medidas para la determinación y establecimiento del perfil de los porteros participantes.

\section{Valoración antropométrica}

Se tuvieron en cuenta los factores antropométricos considerados como importantes para el rendimiento deportivo en el balonmano, siendo la talla, el peso, la masa grasa y la masa magra.

\section{Velocidad}

Test de velocidad con células fotoeléctricas a 5 metros y a 15 metros respecto al punto de salida (Gorostiaga et al., 2005; Haugen et al., 2016; Irigoyen, Vizcay, Pitillas, y Los Arcos Larumbe, 2016; Markoviæ et al., 2015; Vila, Abraldes, y Rodríguez, 2009).

Cada jugador realizó tres intentos con un descanso de 4 minutos siendo el mejor sprint el utilizado para el estudio. La prueba de velocidad mostró un ICC de 0,939 y un CV de 2,4 \% para la medida de $5 \mathrm{~m}$. y un ICC de 0,992 y un CV de 1,19\% para la medida de $15 \mathrm{~m}$.

\section{Tests de salto vertical}

Se realizaron tres tipos de salto con el fin de evaluar la capacidad de salto del jugador de balonmano: SJ, CMJ y ABK (Ferrer-Contreras, 2007; Gorostiaga et al., 2005; Markoviæ et al., 2015; Matthys et al., 2013; Sánchez-Sixto, y Floría, 2017; Valdes, e Irigoyen, 2016). Cada jugador realizó tres intentos por cada modalidad de salto siendo el mejor de cada modalidad el utilizado para el estudio. Los sujetos descansaron 3 minutos entre cada intento con el objetivo de evitar la fatiga (Vila et al., 2012). Se utilizó una plataforma de salto Chronojump Bosco System para calcular la altura mediante el tiempo de vuelo del sujeto. El SJ mostró un ICC de 0,991 y un CV de 4,45 \%, el CMJ mostró un ICC de 0,994 y un CV de 2,61 \%, el ABK mostró un ICC de 0,985 y un CV de $3,45 \%$.

\section{Flexibilidad}

Si relacionamos esta capacidad física con los puestos específicos, cabe decir que sería necesario tener en cuenta que la flexibilidad es mucho más determinante en la demarcación de portero (Srhoj, 2002; Rogulj, Srhoj, Nazor, Srhoj y Cavala, 2005).

Se utilizó la prueba de sit and reach para medir la flexibilidad de la parte baja de la espalda, los extensores de cadera y los músculos flexores de rodilla (Nikolaidis e Ingebrigtsen, 2013). Para ello, se utilizó un cajón sit and reach con una regla milimetrada en su superficie superior externa.

\section{Análisis estadístico}

Se analizó la distribución de las distintas variables de estudio mediante el cálculo de estadísticos descriptivos básicos (media y desviación típica), la distribución de la normalidad (prueba de KolgomorovSmirnov y Lilliefors) y prueba de homogeneidad de Levene.

También se realizó un análisis de varianza de un factor (Anova) y una prueba post-hoc (Tukey) para determinar las posibles diferencias significativas entre las distintas categorías.

La fiabilidad de las distintas pruebas de rendimiento físico se evaluó utilizando el Coeficiente de Correlación Interclase (ICC). Un ICC > 0,75 es considerado muy bueno, $0,41-0,75$ regular-bueno y valores por debajo de 0,40 es considerado bajo y que por tanto dicha prueba de campo es inadecuada (Fleiss, 1986).

El Coeficiente de Variación de la Media (CV) se calculó para cada prueba de campo, siendo inferior al $5 \%$ para cada una. El criterio dep $<$ 0,05 fue utilizado para establecer diferencias significativas.

\section{Resultados}

En las siguientes tablas se muestran los resultados obtenidos en el presente estudio. La prueba de Kolmogorov-Smirnov muestra que todas las variables siguen la normalidad mientras que la prueba de Levenne confirma que se sigue el principio de homogeneidad de la varianza para todas las variables dependientes.

En la tabla 1 se puede observar que las variables y resultados por edades (media y desviación típica), muestran como a medida que aumenta la categoría de los jugadores, lo hacen los valores antropométricos. Además, en las pruebas físicas, mejoran los valores en todas las variables a excepción de la prueba sit and reach. De todas ellas se destaca la

Tabla 1:

Descriptivo básica de las pruebas realizadas.

\begin{tabular}{|c|c|c|c|}
\hline $\begin{array}{l}\text { Prueba } \\
\end{array}$ & $\mathrm{G} 1(\mathrm{M} \pm \mathrm{SD})$ & $\mathrm{G} 2(\mathrm{M} \pm \mathrm{SD})$ & $\mathrm{G} 3(\mathrm{M} \pm \mathrm{SD})$ \\
\hline Peso (kg) & $53,25 \pm 8,04$ & $68,33 \pm 9,91$ & $88,94 \pm 9,32$ \\
\hline Altura (cm) & $160,35 \pm 7,42$ & $172,10 \pm 7,92$ & $183,4 \pm 4,03$ \\
\hline IMC $\left(\mathrm{kg} / \mathrm{m}^{2}\right)$ & $20,70 \pm 2,81$ & $23,12 \pm 3,26$ & $26,58 \pm 2,30$ \\
\hline Grasa corporal (\%) & $12,36 \pm 6,52$ & $12,83 \pm 7,85$ & $16,66 \pm 4,71$ \\
\hline Masa muscular (kg) & $44,03 \pm 6,05$ & $55,73 \pm 7,77$ & $70,18 \pm 4,88$ \\
\hline Sit and reach $(\mathrm{cm})$ & $25,00 \pm 6,39$ & $28,22 \pm 5,83$ & $26,00 \pm 9,38$ \\
\hline Sprint 5m (s) & $1,16 \pm 0,65$ & $1,12 \pm 0,79$ & $1,02 \pm 0,55$ \\
\hline Sprint 15m (s) & $2,88 \pm 0,20$ & $2,68 \pm 0,15$ & $2,48 \pm 0,12$ \\
\hline Salto SJ (cm) & $20,83 \pm 4,23$ & $25,42 \pm 3,24$ & $28,99 \pm 6,03$ \\
\hline Salto CMJ (cm) & $22,82 \pm 4,37$ & $28,04 \pm 3,37$ & $32,12 \pm 6,93$ \\
\hline Salto ABK $(\mathrm{cm})$ & $27,57 \pm 5,31$ & $33,03 \pm 4,57$ & $37,58 \pm 5,85$ \\
\hline \multicolumn{4}{|c|}{ Nota: G1: Grupo de edad 1; G2: Grupo de edad 2; G3: Grupo de edad 3} \\
\hline \multicolumn{4}{|l|}{$\begin{array}{l}\text { Tabla 2: } \\
\text { Índice de s }\end{array}$} \\
\hline Prueba & G1-G2 & G2-G3 & G1-G3 \\
\hline Peso (kg) & $0,002^{*}$ & $0,001^{*}$ & $<0,001 *$ \\
\hline Altura (cm) & $0,003^{*}$ & $0,025^{*}$ & $<0,001 *$ \\
\hline $\operatorname{IMC}\left(\mathrm{kg} / \mathrm{m}^{2}\right)$ & 0,164 & 0,105 & $0,003^{*}$ \\
\hline Grasa corporal (\%) & 0,987 & 0,575 & 0,469 \\
\hline Masa muscular (kg) & $0,001 *$ & $0,002 *$ & $<0,001 *$ \\
\hline Sit and reach $(\mathrm{cm})$ & 0,542 & 0,830 & 0,959 \\
\hline Sprint $5 \mathrm{~m}(\mathrm{~s})$ & 0,478 & $0,039 *$ & $0,003^{*}$ \\
\hline Sprint 15m (s) & 0,054 & 0,140 & $0,001 *$ \\
\hline Salto SJ $(\mathrm{cm})$ & 0,060 & 0,317 & $0,005^{*}$ \\
\hline Salto CMJ $(\mathrm{cm})$ & $0,045^{*}$ & 0,276 & $0,003^{*}$ \\
\hline Salto ABK (cm) & 0,063 & 0,276 & $0,004^{*}$ \\
\hline
\end{tabular}


flexibilidad, una capacidad involutiva que en el caso de los porteros de balonmano debe ser entrenada, pues supone un elemento esencial para poder desarrollar la técnica correctamente, y con ello, detener lanzamientos. Por lo que si son considerados los dos grandes tipos de parada, tanto fragmentaria como explosiva, los elementos que dependen de la elasticidad-flexibilidad son muy importantes.

En cuanto al análisis estadístico ANOVA(Tabla 2), se encontraron diferencias significativas entre Grupo 1 (G1) y Grupo 3 (G3) en ocho de las once variables analizadas, mientras que entre Grupo 1 (G1) y Grupo 2 (G2) y Grupo 2 (G2) y Grupo 3 (G3) sólo se encontraron diferencias significativas en cuatro de las once variables analizadas, la mayoría pertenecientes a variables relacionadas con el perfil antropométrico.

Entre el Grupo 1 (G1) y el Grupo 2 (G2), se establecen diferencias significativas en peso, altura, masa muscular y CMJ. Del mismo modo, entre el Grupo 2 (G2) y el Grupo 3 (G3), se establecen diferencias significativas en peso, altura, masa muscular y sprint 5 metros. Además, entre el Grupo 1 (G1) y el Grupo 3 (G3) existen diferencias significativas en peso, altura, IMC, masa muscular, sprint 5 metros, sprint 15 metros, SJ, CMJ y ABK.

El hecho de que la mayor diferencia se encuentre entre el Grupo 1 (G1) y el Grupo 3 (G3) puede deberse a que son los grupos en los que existe mayor diferencia de edad, y por tanto mayor diferencia a nivel madurativo, influyendo de manera significativa en los resultados antropométricos y de condición física.

Todas estas mejoras evolutivas proveen al portero de una seria de características que son necesarias para el desarrollo de su papel en la portería. Una mayor talla y envergadura se traduce en una mayor cobertura del espacio de la portería, lo cual, al separarse de esta, obliga a los lanzadores a tener que realizar lanzamientos específicos como el liftado o la rosca para poder encontrar los huecos necesarios y así poder puntuar. Del mismo modo la fuerza y la velocidad ayudan en cuanto a la realización de movimientos explosivos que garanticen la interceptación del balón, pues los lanzamientos desde 6 metros son próximos a la colocación del portero, y por tanto, este último, dispone de muy poco tiempo para poder efectuar las acciones y movimientos de parada que los lanzamientos requieran.

\section{Discusión}

De los resultados analizados se puede establecer que el portero se asemeja más a los valores que presentan los jugadores de la primera línea en categorías inferiores, cuya maduración es mayor respecto a las demarcaciones de extremo y pivot. (Matthys et al., 2013). Dichas características correlacionan de forma adecuada con las características antropométricas observadas en los jugadores adultos (Chaouachi et al., 2009; Sporis et al., 2010; Srhoj et al., 2002). No obstante, existen grandes diferencias en las características antropométricas de los jugadores de balonmano adultos según la posición que ocupen en el campo.

Los porteros analizados presentan valores semejantes en estatura, peso, IMC y porcentaje de masa grasa que en estudios realizados con anterioridad en este tipo de población (Grijota et al., 2012; Matthys et al., 2013; Visnapu y Jürimäe, 2009). Si bien es cierto que el Grupo 3 (G3) presenta valores inferiores en cuanto a altura y peso, puede deberse a que fue comparado con jugadores de categoría absoluta con un desarrollo físico completo, al que dicho grupo todavía no ha llegado.

Las principales diferencias entre grupos, sobre todo entre el Grupo 1 (G1) y el resto, las encontramos en las variables relacionadas con el perfil antropométrico, puesto que es a partir de los 14-15 años en los chicos y los 12 en chicas cuando se produce el pico de velocidad de crecimiento en estatura (Beunen y Malina, 1987). En relación con lo explicado anteriormente, Lidor et al. (2005) realizó un estudio en el que no encontró diferencias en la altura ni en la masa corporal en jugadores de balonmano de edades comprendidas entre los 12 y los 14 años.

Concretamente, en este estudio el análisis preliminar de los resultados muestra que las pruebas físicas mejoran conforme aumenta la categoría de los deportistas, debido principalmente a la maduración de los jugadores y al aumento del volumen de trabajo realizado a lo largo de los años (Vaeyens, Lenoir, Williams, y Philippaerts, 2008).

$\mathrm{El}$ hecho de que no se encuentren diferencias significativas en el rendimiento deportivo entre el Grupo 2 (G2) y el Grupo 3 (G3), puede deberse a su estabilización a partir de la pubertad (Vila, Fernández, y Rodríguez, 2007) disminuyéndose las diferencias en la condición física conforme aumenta la edad (Grijota et al., 2012). Por tanto, parecen coherente los resultados obtenidos y más si tenemos en cuenta otros estudios en los que se puede ver como los jugadores de balonmano de categorías inferiores tienen una maduración biológica más temprana respecto a los sujetos de su misma edad (Matthys, Vaeyens, Coelho e Silva, Lenoir, y Philippaerts, 2012; Pearson, Naughton, y Torode, 2006) y respecto a otros deportes (Grijota et al., 2012).

Por lo que respecta a la capacidad de salto y velocidad de desplazamiento, podemos ver como los resultados se asemejan a los obtenidos en el estudio de Matthys et al. (2013) para la demarcación de portero en el que estos presentan valores bajos en las distintas pruebas físicas junto a los pivotes.

Los jugadores de balonmano suelen presentar valores bastante bajos en flexibilidad si los comparamos con otros deportes (Grijota et al., 2012). No obstante, no ocurre lo mismo con los porteros de balonmano, pudiéndose observar en los jugadores analizados unos valores altos puesto que se trata de una cualidad muy importante en las acciones de los porteros (Srhoj, 2002; Rogulj, Srhoj, Nazor, Srhoj y Cavala, 2005) que los diferencia del resto de posiciones. El hecho de que no encontremos un incremento significativo en esta cualidad conforme aumenta la categoría, como si ocurre en el resto de cualidades físicas, puede deberse según Grosser y Müller (1992) a que los periodos de gran flexibilidad se mantienen hasta los doce años, a partir de edad, la flexibilidad evolucionará de una forma negativa, haciéndose cada año más limitada, como consecuencia de la estabilización del esqueleto y al aumento de la hipertrofia muscular debido a la liberalización de andrógenos y estrógenos.

Finalmente cabe decir a partir de todo lo expuesto que elegir a los jugadores por su maduración biológica puede ser un error ya que no todos los niños se desarrollan al mismo tiempo, pudiendo incluso invertirse dicha situación durante la adolescencia (Pearson et al., 2006; Vaeyens, Lenoir, Williams, y Philippaerts, 2008). De esta forma, estaríamos excluyendo a muchos deportistas que tienen una maduración más tardía que aun teniendo unas buenas cualidades para el balonmano se ven limitadas por aspectos físicos. Al mismo tiempo, debido a la especialización temprana, estaríamos encasillando a los jóvenes deportistas en una posición para la que están físicamente más dotados sin tener en cuenta la posible evolución que puedan tener y que les haría rendir más en otros puestos.

\section{Conclusiones}

En cuanto a las conclusiones que establece el presente trabajo sobre el perfil condicional y antropométrico de porteros de balonmano de categorías inferiores, se destaca en un primer lugar que el portero de balonmano posee un nivel madurativo mayor que al de otros perfiles de deportistas que practican otros deportes. En relación a este hecho y a las demandas físicas de este puesto, se muestra un porcentaje de grasa mayor en relación a otras posiciones de juego. Por otro lado, por lo que respecta a los valores condicionales, se destaca sobre todo los resultados alcanzados en flexibilidad frente a otras cualidades físicas. Finalmente, se establecen como cualidades diferenciadores entre grupos de edad, las relacionadas con el perfil antropométrico más que al condicional.

\section{Referencias}

Beunen, G., y Malina, R. M. (1987). Growth and biologic maturation: relevance to athletic performance. En Bar-Or (ed). Advances in Pediatric Sport Sciences (1-19). Champaign, IL: Human Kinetics. Chaouachi, A., Brughelli, M., Levin, G., Boudhina, N. B., Cronin, J., y Chamari, K. (2009). Anthropometric, physiological and perfor- 
mance characteristics of elite team-handball players. Journal of Sports Sciences, 27, 151-157.

Díaz, J. J., Rojas, W. S., y Castro, M. M. (2016). Uso de practica en bloque y aleatoria en el desempeño de lanzar por encima del hombro en adultos. Retos: nuevas tendencias en educación física, deporte y recreación, (29), 9-12.

Dumitru, D. C. (2010). The importance of a specific warm-up on the performance of the handball goalkeeper. Journal of Physical Education \& Sport, 28(3), 23-31.

Espina-Agulló, J. J., Pérez-Turpin, J. A., Jiménez-Olmedo, J. M., Penichet-Tomás, A., y Pueo, B. (2016). Effectiveness of Male Handball Goalkeepers: A historical overview 19822012. International Journal of Performance Analysis in Sport, 16(1), 143-156.

Ferrer-Contreras, M.C. (2007). Efectos de dos métodos de entrenamiento de fuerza sobre el índice de Bosco en jugadoras de balonmano de División de Honor. Effects of two strength training methods in Bosco Index on elite handball female player. Retos: nuevas tendencias en Educación Física, Deporte y Recreación, (11), 33-36.

Fleiss, J.L. (1986). The Design and Analysis of Clinical Experiments. New York: John Wiley \& Sons.

Gorostiaga, E. M., Granados, C., Ibáñez, J., e Izquierdo, M. (2005). Differences in physical fitness and throwing velocity among elite and amateur male handball players. International Journal of Sports Medicine, 26, 225-232.

Grijota, J., Muñoz, D., Crespo, C., Robles, M. C., y Maynar, M. (2012). Análisis comparativo de la composición corporal y de la condición física en deportistas en edad escolar practicantes de balonmano, natación y kárate. Revista Kronos, 11(1).

Grosser, M., y Müller, H. (1992). Desarrollo muscular: un nuevo concepto de musculación ( «Power Stretch»). Barcelona, Hispano Europea.

Haugen, T. A., Tønnessen, E., y Seiler, S. (2016). Physical and physiological characteristics of male handball players: influence of playing position and competitive level. The Journal of Sports Medicine and Physical Fitness.

Irigoyen, J. Y., Vizcay, J. J., Pitillas, I., y Los Arcos Larumbe, A. (2016). Diferencias entre alumnos de una escuela de atletismo y estudiantes de educación primaria en la capacidad de aceleración y cambio de dirección. Retos: nuevas tendencias en educación física, deporte y recreación, (29), 28-31.

Iturriaga, F. M.A., García-Cervantes, L., y Lara, E. R. (2016). Factores asociados a la eficacia de gol en waterpolo. Retos: nuevas tendencias en educación física, deporte y recreación, (29), 105-108.

Lidor, R., Falk, A., Arnon, M., Cohen, Y., Segal, G., y Lander, Y.(2005). Measurement of talent in team handball: The questionable use of motor and physical tests. Journal of Strength and Conditioning Research, 19, 318-325.

Markovic, S., Vuckovic, I., Sekulc, ž, y Gadžic, A. (2015). Physical performance modeling among young basketball and handball players. Facta Universitatis, Series: Physical Education and Sport, 13(2), 263-273.

Matthys, S. P., Fransen, J., Vaeyens, R., Lenoir, M., y Philippaerts, R. (2013). Differences in biological maturation, anthropometry and physical performance between playing positions in youth team handball. Journal of Sports Sciences, 31(12), 1344-1352.

Matthys, S. P. J., Vaeyens, R., Coelho e Silva, M. J., Lenoir, M., y Philippaerts, R. M. (2012). The contribution of growth and maturation in the functional capacity and skill performance of male adolescent handball players. International Journal of Sports Medicine, 33, 543-549.

Milanese, C., Piscitelli, F., Lampis, C., y Zancanaro, C. (2011). Anthropometry and body composition of female handball players according to competitive level or the playing position. Journal of Sports Sciences, 29(12), 1301-1309.

Mohamed, H. S., Vaeyens, R., Matthys, S., Multael, M., Lefevre, J., Lenoir, M., y Philippaerts, R. M. (2009). Anthropometric and performance measures for the development of a talent detection and identification model in youth handball. Journal of Sports Sciences, 27, 257-266.

Nikolaidis, P. T., e Ingebrigtsen, J. (2013). Physical and physiological characteristics of elite male handball players from teams with a different ranking. Journal of Human Kinetics, 38, 115-124.

Pearson, D. T., Naughton, G. A., y Torode, M. (2006). Predictability of physiological testing and the role of maturation in talent identification for adolescent team sports. Journal of Science and Medicine in Sport, 9, 277-287.

Peña, J., Moreno-Doutres, D., Coma, J., Cook, M., y Buscà, B. (2016). Anthropometric and fitness profile of high-level basketball, handball and volleyball players. Revista Andaluza de Medicina del Deporte.

Rogulj, N., Srhoj, V., Nazor, M., Srhoj, L., y Èavala, M. (2005). Some anthropologic characteristics of elite female handball players at different playing positions. Collegium Antropologicum, 29(2), 705709.

Sánchez-Sixto, A., y Floría, P. (2017). Efecto del entrenamiento combinado de fuerza y pliometría en variables biomecánicas del salto vertical en jugadoras de baloncesto. Effects of combined plyometric and resistance training in biomechanical variables of the vertical jump in basketball players. Retos: nuevas tendencias en educación física, deporte y recreación, (31), 114-117.

Srhoj, V., Marinovic, M., y Rogulj, N. (2002). Position specific morphological characteristics of top-level male handball players. Collegium Antropologicum, 26, 219-227.

Sporis, G., Vuleta, D., Vuleta, D. J., y Milanovic, D. (2010). Fitness profiling in handball: Physical and physiological characteristics of elite players. Collegium Antropologicum, 34, 1009-1014.

Vaeyens, R., Lenoir, M., Williams, A. M., y Philippaerts, R. M. (2008). Talent identification and development programmes in sport-Current models and future directions. Sports Medicine, 38, 703-714.

Valdes, P., e Irigoyen, J. Y.(2016). Análisis de la condición física, tipo de actividad física realizada y rendimiento académico en estudiantes de educación secundaria. Retos: nuevas tendencias en educación física, deporte y recreación, (30), 64-69.

Vila, H., Abraldes, J. A., y Rodríguez, N. (2009). Estudio del perfil antropométrico del jugador juvenil de balonmano en la Región de Murcia. Retos: nuevas tendencias en educación física, deporte y recreación, (16), 80-85.

Vila H., Fernández J.J.y Rodríguez F. (2007). Evolución de la condición física en jugadoras de balonmano en las categorías infantil, cadete y juvenil. Apunts. Educación física y deportes, 1(87), 99-106.

Vila, H., Manchado, C., Rodriguez, N., Abraldes, J.A., Alcaraz, P. E., y Ferragut, C. (2012). Anthropometric profile, vertical jump, and throwing velocity in elite female handball players by playing positions. The Journal of Strength \& Conditioning Research, 26(8), 2146-2155.

Visnapuu, M., y Jürimäe, T. (2009). Relations of anthropometric parameters with scores on basic and specific motor tasks in young handball players. Perceptual and motor skills, 108(3), 670-676.

Ziv, G., y Lidor, R. (2009). Physical characteristics, physiological attributes, and on-court performances of handball players: Areview. European Journal of Sport Science, 9, 375-386.

Zurgo, T., de Haro, V. M., Hernández, M. T. L., y Arribas, I. S. (2016). Velocidad de crecimiento de deportistas adolescentes tecnificados de natación, waterpolo, saltos y natación sincronizada. Retos: nuevas tendencias en educación física, deporte y recreación, (30), 98100.

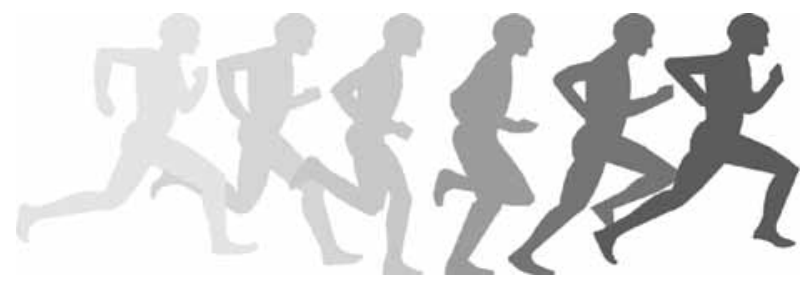

